\title{
On the Separation of Lorentz and Magnetization Forces in the Transduction Mechanism of Electromagnetic Acoustic Transducers (EMATs)
}

\author{
Matthias Seher $^{1}$ and Peter B. Nagy ${ }^{1,2, *}$ \\ ${ }^{1}$ Department of Mechanical Engineering, Imperial College London, London, UK \\ ${ }^{2}$ Department of Aerospace Engineering and Engineering Mechanics, \\ University of Cincinnati, Cincinnati, Ohio 45221, USA
}

\begin{abstract}
Electromagnetic Acoustic Transducers (EMATs) are widely used in Nondestructive Evaluation (NDE) of metals, including the inspection of ferritic steels. Optimization of EMATs in ferromagnetic materials is often accomplished using computational simulations that account for the three main types of transduction mechanism, namely the effects of Lorentz forces, magnetization forces, and magnetostriction. The extensive literature on the Lorentz and magnetization mechanisms of EMATs has been historically scattered with alternative notations causing conflicting interpretations of the fundamental governing equations that often led to erroneous implementations of these equations in computational simulations. The purpose of this paper is to clarify this issue. The confusion is caused by the arbitrary nature of the separation of electromagnetic transduction force into Lorentz and magnetization components. This paper shows that apparently contradicting models are actually equivalent if the Lorentz and magnetization forces are chosen to correctly match each other, while mixing otherwise acceptable but mutually incompatible terms leads to unacceptable modelling errors. This paper also provides guidelines for the implementation of these transduction mechanisms in computational models for EMATs.
\end{abstract}

Keywords: Electromagnetic Acoustic Transducers; transduction; Lorentz force; magnetization force.

*Corresponding author. Tel.: +1-513-556-3353; E-mail address: peter.nagy@ uc.edu (P.B. Nagy) 


\section{Introduction}

Electromagnetic Acoustic Transducers (EMATs) are widely used in the field Nondestructive Evaluation (NDE) for the generation and detection of elastic waves to perform a variety of inspection tasks. For many decades, EMATs have been commercially exploited by various manufacturers and the complex transduction phenomena underlying their operation have been the subject of academic research aimed mainly at optimizing their design [1]. Generally, EMATs consist of two main components, a permanent magnet and an induction coil. The permanent magnet generates a static bias magnetic field, which penetrates into the specimen while the excitation current flowing in the coil induces eddy currents in the surface region of the specimen within the electromagnetic skin depth. The secondary eddy current is a mirror image of the primary coil current but flows in the opposite direction in accordance with Lenz's law. In transmission, the interaction between the static and dynamic magnetic fields gives rise to generation of elastic waves. In reception, the elastic waves generate an electromotive force which induces a voltage in the sensing coil that can be measured at the terminals of the coil.

One drawback of EMATs is that, due to the need for generating eddy currents, they are generally limited to operation in conducting materials, such as metals, though operation based on magnetization and magnetostriction in non-conducting magnetic materials, such as ferrites, is also feasible. In the case of ferromagnetic materials with high conductivity, such as steel, there are three major transduction mechanisms that should be accounted for [2]. First, the magnetic Lorentz force that acts on moving charges. The second transduction mechanism is the magnetization force that can be understood as the force acting on magnetic moments in the presence of a spatially varying magnetic field. The third mechanism is magnetostriction that can be described by a constitutive relationship between the magnetostrictive eigenstrain and the magnetizing field that generally exhibits vast uncertainties in the properties of the material [3]. Magnetostriction will not be included in the following discussion that is aimed at the Lorentz and magnetization mechanisms only.

Historically, much of the extensive literature on the transduction mechanisms of EMATs, which dates back to the 1970s (see references in [1]), contains multiple notations of the fundamental governing equations, some of which are conflicting representations and may lead to erroneous approximations of the transduction mechanisms. This may lead to problems in modelbased EMAT design as the sensor's performance may not match experimental results, leading to 
significant increase in development time due to repeated iterations of experimental tests and design reviews. The confusion between multiple representations arises mainly because of the arbitrary separation of the electromagnetic transduction force into Lorentz and magnetization components. The aim of this paper is to address the issue of conflicting representations of the EMAT transduction process due to this arbitrary separation. It will be shown that different models are actually equivalent if the Lorentz and magnetization forces are chosen to correctly match each other, while mixing otherwise acceptable but mutually incompatible terms leads to unacceptable modelling errors. First, the various transduction models found in the literature will be reviewed. Later, it will be illustrated through numerical simulations of both longitudinal and shear wave EMATs that improper separation of the transduction force into Lorentz and magnetization components leads to unacceptable modelling errors.

\section{EMAT Transduction}

The magnetic flux density $\mathbf{B}=\mu_{0}(\mathbf{H}+\mathbf{M})$ is proportional to the sum of the magnetic field $\mathbf{H}$ and the magnetization $\mathbf{M}$, where $\mu_{0}$ is the magnetic permeability of free space. For the purposes of calculating the magnetic forces acting on elastic solid bodies, it is customary to separate the magnetic flux density $\mathbf{B}$ into external and internal parts [4-6] both of which might have static and dynamic components

$$
\mathbf{B}=\mathbf{B}^{\mathrm{e}}+\mathbf{B}^{\mathrm{i}}=\overline{\mathbf{B}}^{\mathrm{e}}+\overline{\mathbf{B}}^{\mathrm{i}}+\tilde{\mathbf{B}}^{\mathrm{e}}+\tilde{\mathbf{B}}^{\mathrm{i}} .
$$

Here and everywhere else in this paper, bars and tildes over variables indicate static and dynamic components. Only harmonic time dependence will be considered and the tilde sign will refer to the fundamental harmonic only. The external field is produced by sources external to the body under consideration. It represents the magnetic field in the absence of the body, i.e., what the field would be without conductivity and magnetization in the body. In the case of EMATs, $\overline{\mathbf{B}}^{\mathrm{e}}=\mu_{0} \overline{\mathbf{H}}^{\mathrm{e}}$ is the static bias field produced by either a permanent magnet or a direct current (dc) electromagnet in air and $\tilde{\mathbf{B}}^{\mathrm{e}}=\mu_{0} \tilde{\mathbf{H}}^{\mathrm{e}}$ is the dynamic excitation field produced by the alternating current (ac) flowing in the coil of the EMAT also in air. The internal field is produced by sources internal to the body due to the conductivity and magnetic susceptibility of the material in addition to the external field. These internal sources are the eddy currents and aligned magnetic dipoles inside the volume of the body. Specifically, $\overline{\mathbf{B}}^{\mathrm{i}}=\mu_{0} \overline{\mathbf{M}}$ is the static 
internal field produced by the static external field via magnetization in the test object and $\tilde{\mathbf{B}}^{\mathrm{i}}=\mu_{0}\left(\tilde{\mathbf{H}}^{\mathrm{i}}+\tilde{\mathbf{M}}\right)$ is the dynamic internal field produced by the dynamic external field partly via induction of eddy currents and partly via magnetization of the material.

Similarly, the transduction force $\mathbf{F}$ acting on the material can be separated into external $\mathbf{F}^{\mathrm{e}}$ and internal $\mathbf{F}^{\mathrm{i}}$ parts both of which might have static and alternating components $\mathbf{F}=\mathbf{F}^{\mathrm{e}}+\mathbf{F}^{\mathrm{i}}=\overline{\mathbf{F}}^{\mathrm{e}}+\overline{\mathbf{F}}^{\mathrm{i}}+\tilde{\mathbf{F}}^{\mathrm{e}}+\tilde{\mathbf{F}}^{\mathrm{i}}$.

External forces are those whose reaction forces are outside the body, which in the case under consideration means components of the EMAT. Specifically, the reaction force of the static external force $\overline{\mathbf{F}}^{\mathrm{e}}$ is acting on the permanent magnet or dc electromagnet producing the static bias field and the reaction force of the dynamic external force $\tilde{\mathbf{F}}^{\mathrm{e}}$ is acting on either the biasing magnet or the coil producing the dynamic excitation field. Internal forces are forces acting on a part of the material that are counterbalanced by reaction forces acting on another part of the material. When the total transduction force acting over the whole interaction volume of the material is calculated the total internal force vanishes $\mathbf{F}^{\mathrm{i}}=\mathbf{0}$ so that the total transduction force reduces to the total external force [5]

$\mathbf{F}=\mathbf{F}^{\mathrm{e}}$.

Generally, although internal forces always cancel each other in the sense that they cannot cause overall acceleration of the body, they can still cause significant deformations in it.

The electromagnetic interaction volume of EMATs is restricted to a usually shallow surface layer that is limited by the electromagnetic skin depth

$$
\delta=\frac{1}{\sqrt{\pi f \mu \sigma}},
$$

where $f$ is the inspection frequency, $\mu$ is the magnetic permeability, and $\sigma$ is the electric conductivity of the material. In most EMAT applications, the electromagnetic skin depth $\delta$ is much smaller than the wavelength $\lambda$ of the acoustic wave produced. One practical consequence of this is that both types of interaction forces and even magnetostrictive strains can be accurately represented by equivalent surface tractions applied to the surface of the body that would be otherwise traction free [3,7-11]. For our immediate purposes it is even more important that when $\delta<<\lambda$ internal forces can be neglected in modeling the transduction mechanism of EMATs. 
Although internal forces can cause weak deformations in the material, the balancing counter forces are acting in the very close vicinity of each other so that the resulting deformations are weak compared to those produced by external forces. Therefore, in the remainder of the paper the term transduction force will refer to the external transduction force only.

The magnetic transduction force $\mathbf{F}$ is routinely, albeit somewhat arbitrarily, written as the sum of the Lorentz force $\mathbf{F}^{\mathrm{L}}$ and the magnetization force $\mathbf{F}^{\mathrm{m}}$

$\mathbf{F}=\mathbf{F}^{\mathrm{L}}+\mathbf{F}^{\mathrm{m}}=\int_{V} \mathbf{f}^{\mathrm{L}} d V+\int_{V} \mathbf{f}^{\mathrm{m}} d V$,

where $\mathbf{f}^{\mathrm{L}}$ and $\mathbf{f}^{\mathrm{m}}$ are Lorenz and magnetization body forces, respectively, that are integrated over the interaction volume $V$. For a continuous charge distribution in motion, the general Lorentz body force equation can be written as follows

$\mathbf{f}^{\text {Lorentz }}=\rho(\mathbf{E}+\mathbf{v} \times \mathbf{B})$,

where $\rho$ denotes the charge density (charge per unit volume), $\mathbf{E}$ is the electric field, $\mathbf{B}$ is the magnetic flux density, and $\mathbf{v}$ is the charge velocity. In EMAT terminology, the Lorentz force is meant only to refer to the second term of magnetic origin in Eq. (6), which can be conveniently written in terms of the conduction current density $\mathbf{J}^{\mathfrak{c}}=\rho \mathbf{v}$ as

$\mathbf{f}^{\mathrm{L}}=\mathbf{J}^{\mathrm{c}} \times \mathbf{B}$.

Following Brown's rigorous derivation of the total electromagnetic force acting on a conductive magnetizable body [5], the external Lorentz body force is

$\mathbf{f}^{\mathrm{L}}=\mathbf{J}^{\mathrm{c}} \times \mathbf{B}^{\mathrm{e}}$

and the magnetization body force can be calculated from

$\mathbf{f}^{\mathrm{m}}=\mu_{0} \mathbf{M} \cdot \nabla \mathbf{H}^{\mathbf{e}}$,

where $\mathbf{M}$ is the magnetization in the material. To be specific, Eqs. (8) and (9) correspond to Eq. (5.3) and the physical interpretation given in the following paragraph on page 55 of [5].

While there is a complete agreement in the literature on these fundamental transduction equations of EMATs in ferromagnetic materials (without the effect of magnetostriction), there are two important points over which different interpretations have been proposed. The first point of contention concerns the interpretation of the Lorentz body force while the second one concerns the dyadic product that appears in the magnetization body force. Brown was clear that the total Lorentz force should be calculated from the external magnetic field following Eq. (8) as 
$\mathbf{f}^{\mathrm{L} 1}=\mu_{0} \mathbf{J}^{\mathrm{c}} \times \mathbf{H}^{\mathrm{e}}$

and the total magnetization body force given in Eq. (9) should be interpreted as

$\mathbf{f}^{\mathrm{m} 1}=\mu_{0}(\mathbf{M} \cdot \nabla) \mathbf{H}^{\mathbf{e}} \quad$ or $\quad f_{i}^{\mathrm{m} 1}=\mu_{0} M_{j} H_{i, j}^{\mathrm{e}}$.

Here, the parenthesis was added to the equation of the magnetization body force which was also spelled it out in indicial notation to make the notation absolutely unequivocal. Also, in order to distinguish between different interpretations of the Lorentz and magnetization body forces, the number 1 was added to their superscripts to indicate the original interpretation of Brown [5].

It should be mentioned that Brown also recognized that in some cases it might be necessary to express the magnetization force in terms of the total magnetic field $\mathbf{H}=\mathbf{H}^{\mathrm{e}}+\mathbf{H}^{\mathrm{i}}$ rather than in terms of the external field $\mathbf{H}^{\mathrm{e}}$ only. The Lorentz body force is essentially unaffected by this change of variable as Eq. (10) can be formally rewritten as follows

$\mathbf{f}^{\mathrm{L} 2}=\mu_{0} \mathbf{J}^{\mathrm{c}} \times \mathbf{H}$.

Strictly speaking, $\mathbf{f}^{\mathrm{L} 2}$ is not identical to $\mathbf{f}^{\mathrm{L} 1}$ as previously given in Eq. (10), but their dynamic components of interest in our discussion are identical since eddy currents have only dynamic components and $\tilde{\mathbf{f}}^{\mathrm{L} 1}=\mu_{0} \tilde{\mathbf{J}}^{\mathrm{c}} \times \overline{\mathbf{H}}^{\mathrm{e}}$ is identical to $\tilde{\mathbf{f}}^{\mathrm{L} 2}=\mu_{0} \tilde{\mathbf{J}}^{\mathrm{c}} \times \overline{\mathbf{H}}$ simply because the static field is produced entirely by external sources, i.e., $\overline{\mathbf{H}}=\overline{\mathbf{H}}^{\mathrm{e}}$. In contrast, $\tilde{\mathbf{H}} \neq \tilde{\mathbf{H}}^{\mathrm{e}}$ because of the dynamic magnetic field generated by eddy currents in conducting materials. In Eq. (12) and the rest of the paper the number 2 was added to superscripts to indicate the alternative full-field formulation of Brown [5].

In terms of the total magnetic field $\mathbf{H}$, the total magnetization force can be written as

$\mathbf{F}^{\mathrm{m} 2}=\int_{V} \mathbf{f}^{\mathrm{m} 2} d V+\int_{S} \mathbf{T}^{\mathrm{m} 2} d S$,

where $\mathbf{n}$ is the outgoing normal unit vector of the surface $S$ that encloses volume $V$. Here, the magnetization body force can be written with the total magnetic field $\mathbf{H}$ as follows

$\mathbf{f}^{\mathrm{m} 2}=\mu_{0}(\mathbf{M} \cdot \nabla) \mathbf{H} \quad$ or $\quad f_{i}^{\mathrm{m} 2}=\mu_{0} M_{j} H_{i, j}$.

The magnitude of the normal magnetization surface force in Eq. (13) is

$\mathbf{T}^{\mathrm{m} 2}=\frac{1}{2} \mu_{0} M_{\mathrm{n}}^{2} \mathbf{n}$,

where $M_{\mathrm{n}}$ denotes the normal magnetization of the material at the surface and $\mathbf{n}$ is the normal unit vector of the surface. Eqs. (13), (14) and (15) correspond to Eq. (5.15) on page 57 in [5]. 
In computational simulations using finite element (FE) or other numerical methods the external field $\mathbf{H}^{\mathrm{e}}$ can be readily calculated separately from the total field $\mathbf{H}$, therefore the simpler formulation of $\mathbf{f}^{\mathrm{m} 1}$ given in Eq. (11) might be preferred since it does not require the additional surface integration as the corresponding surface traction vanishes, i.e., $\mathbf{T}^{\mathrm{m} 1}=\mathbf{0}$. Still, Eq. (13) is worth considering because it is often used in the literature to calculate the magnetization force in EMAT simulations. It will be demonstrated later that the external-field $\left(\mathbf{F}^{\mathrm{ml}}\right)$ and total-field $\left(\mathbf{F}^{\mathrm{m} 2}\right.$ and $\left.\mathbf{T}^{\mathrm{m} 2}\right)$ versions of the magnetization forces are equivalent in the sense that they give identical results in the low-frequency approximation $(\delta<<\lambda)$ when the somewhat different spatial distribution of the applied transduction forces makes little or no difference. At higher frequencies these two methods still give qualitatively very similar results with some minor differences that increase with frequency as the differences in the spatial distributions of the transduction forces start to exhibit themselves. In contrast, the above mentioned differences in the interpretation of these transduction equations refer to far more significant disparities that, if used incorrectly, might lead to significant errors in EMAT simulations.

The first point of contention mentioned above is related to the common interpretation of the Lorentz body force as being proportional to the total magnetic flux density $\mathbf{B}$ through $\mathbf{f}^{\mathrm{L} 3}=\mathbf{J}^{\mathrm{c}} \times \mathbf{B}=\mu_{0} \mathbf{J}^{\mathrm{c}} \times(\mathbf{H}+\mathbf{M})$

Although calculating the Lorentz body force this way is perfectly correct from a physical point of view, this interpretation of the Lorentz body force includes internal forces proportional to $\mathbf{M}$ that are cancelled by internal magnetization forces acting on the aligned magnetic dipoles of the material. Thompson was the first to argue that as the moving electrons collide with the lattice and pass on their momentum to it, these internal forces cancel each other in the transduction mechanism of EMATs [12], therefore Brown's interpretation given in Eq. (10) is the right one unless the counterbalancing magnetization force is also included in the calculation.

The second point of contention in the literature is related to the proper interpretation of the dyadic product that appears in the magnetization body force given in Eq. (9). The dyadic interpretation of the gradient $\mathbf{G}=\nabla \mathbf{H}$ of the magnetic vector field $\mathbf{H}$ is based on the standard tensor product $\mathbf{G}=\nabla \otimes \mathbf{H}$, or $G_{i j}=H_{j, i}$ in indicial notation, so that $\mathbf{f}^{\mathrm{m} 3}=\mu_{0} \mathbf{M} \cdot \nabla \mathbf{H}=\mu_{0} \mathbf{M} \cdot(\nabla \mathbf{H}) \quad$ or $\quad f_{i}^{\mathrm{m} 3}=\mu_{0} M_{j} H_{j, i}$. 
However, Brown's original interpretation for the gradient $\mathbf{G}=\nabla \mathbf{H}$ of the magnetic vector field $\mathbf{H}$ was $G_{i j}=H_{i, j}$ so that

$\mathbf{f}^{\mathrm{m} 2}=\mu_{0} \mathbf{M} \cdot \nabla \mathbf{H}=\mu_{0}(\mathbf{M} \cdot \nabla) \mathbf{H} \quad$ or $\quad f_{i}^{\mathrm{m} 2}=\mu_{0} M_{j} H_{i, j}$.

The notation used by Brown [5] is the same interpretation of the gradient of a vector as it is given in many contemporary textbooks on tensor notation, such as $[13,14]$, although there are also textbooks, for example [15], that use the alternative dyadic interpretation. Unfortunately, these two different notations lead to physically very different results when applied to rotational fields like $\mathbf{H}$ whose curl does not vanish when $\nabla \times \mathbf{H}=\mathbf{J} \neq 0$. This means that the dyadic interpretation of the gradient tensor can be applied to Brown's results on the magnetization force only when the calculation is based on the external magnetic field because then $\nabla \times \mathbf{H}^{\mathrm{e}}=0$, therefore $(\mathbf{M} \cdot \nabla) \mathbf{H}^{\mathrm{e}}=\mathbf{M} \cdot\left(\nabla \mathbf{H}^{\mathrm{e}}\right)$. However, the dyadic interpretation of the gradient tensor leads to erroneous results when it is applied to Brown's results on the magnetization force based on the total magnetic field $\mathbf{H}$ because then $\nabla \times \mathbf{H} \neq 0$, therefore $(\mathbf{M} \cdot \nabla) \mathbf{H} \neq \mathbf{M} \cdot(\nabla \mathbf{H})$.

Interestingly, these two apparently unrelated discrepancies between the Lorentz forces $\Delta \mathbf{f}^{\mathrm{L}}$ on one side and the magnetization forces $\Delta \mathbf{f}^{\mathrm{m}}$ on the other side exactly cancel each other. From Eqs. (12) and (16)

$$
\Delta \mathbf{f}^{\mathrm{L}}=\mathbf{f}^{\mathrm{L} 3}-\mathbf{f}^{\mathrm{L} 2}=\mathbf{J}^{\mathrm{c}} \times \mathbf{B}-\mu_{0} \mathbf{J}^{\mathrm{c}} \times \mathbf{H}=\mu_{0} \mathbf{J}^{\mathrm{c}} \times \mathbf{M},
$$

while from Eqs. (14) and (17)

$$
\Delta \mathbf{f}^{\mathrm{m}}=\mathbf{f}^{\mathrm{m} 3}-\mathbf{f}^{\mathrm{m} 2}=\mu_{0} \mathbf{M} \cdot(\nabla \mathbf{H})-\mu_{0}(\mathbf{M} \cdot \nabla) \mathbf{H} .
$$

After some tedious tensor algebra, it can be shown that $(\mathbf{M} \cdot \nabla) \mathbf{H}-\mathbf{M} \cdot(\nabla \mathbf{H})=(\nabla \times \mathbf{H}) \times \mathbf{M}$, therefore Eq. (20) can be transformed into

$$
\Delta \mathbf{f}^{\mathrm{m}}=-\mu_{0} \mathbf{J}^{\mathrm{c}} \times \mathbf{M}=-\Delta \mathbf{f}^{\mathrm{L}},
$$

where it was exploited that $\nabla \times \mathbf{H}=\mathbf{J}^{\mathrm{c}}$. In other words, $\mathbf{f}^{\mathrm{L} 2} \neq \mathbf{f}^{\mathrm{L} 3}$ and $\mathbf{f}^{\mathrm{m} 2} \neq \mathbf{f}^{\mathrm{m} 3}$, but $\mathbf{f}^{\mathrm{L} 2}+\mathbf{f}^{\mathrm{m} 2}=\mathbf{f}^{\mathrm{L} 3}+\mathbf{f}^{\mathrm{m} 3}$.

Of course, this apparently fortuitous cancellation of the differences between methods \#2 and \#3 is really not a coincidence at all. In addition to the dynamic Lorentz force $\tilde{\mathbf{f}}^{\mathrm{L} 1}$ produced by the external field only, $\tilde{\mathbf{f}}^{\mathrm{L} 2}$ also includes the internal Lorentz force due to the static material magnetization field $\overline{\mathbf{M}}$ acting on the conduction current $\tilde{\mathbf{J}}^{\mathrm{c}}$. As Thompson argued [12], this 
internal force also produces a counterforce of equal magnitude but opposite sign on the lattice that is a magnetization force due to the dynamic component of the internal magnetic field $\tilde{\mathbf{H}}^{\mathrm{i}}$ of the eddy current acting on the static part of the magnetization $\overline{\mathbf{M}}$. In other words, adding the internal Lorentz force $\mu_{0} \tilde{\mathbf{J}}^{\mathrm{c}} \times \overline{\mathbf{M}}$ to the external Lorentz force $\mathbf{f}^{\mathrm{L} 2}$ and then subtracting the same internal force $\mu_{0} \tilde{\mathbf{J}}^{\mathrm{c}} \times \overline{\mathbf{M}}$ from the external magnetization force $\mathbf{f}^{\mathrm{m} 2}$ does not affect the total transduction force. From a practical point of view, removing the internal magnetic field $\tilde{\mathbf{H}}^{\mathrm{i}}$ of the eddy current from the total magnetic field $\mathbf{H}$ renders it non-rotational and therefore insensitive to the way how the tensor gradient is calculated. From a conceptual point of view, the equivalence of methods \#2 and \#3 illustrates that the somewhat arbitrary separation of the total transduction force into Lorentz and magnetization forces is really only a question of semantics since a component of the Lorentz force is necessarily the reaction force of a component of the magnetization force.

Finally, a fourth benchmark method based on Maxwell's stress tensor is suggested as the ultimate tool for avoiding the above described issues caused by the arbitrary separation of transduction body force into Lorentz and magnetization components. The electromagnetic body force acting on conductive magnetizable materials can be derived from Maxwell's famous field equations as (see, for example, [16])

$$
\begin{aligned}
\mathbf{f}^{\mathrm{M}} & =\varepsilon_{0}[(\nabla \cdot \mathbf{E}) \mathbf{E}-(\mathbf{E} \cdot \nabla) \mathbf{E}]+\frac{1}{\mu_{0}}[(\nabla \cdot \mathbf{B}) \mathbf{B}-(\mathbf{B} \cdot \nabla) \mathbf{B}] \\
& -\frac{1}{2} \nabla\left[\varepsilon_{0} \mathbf{E} \cdot \mathbf{E}+\frac{1}{\mu_{0}} \mathbf{B} \cdot \mathbf{B}\right]-\varepsilon_{0} \frac{\partial}{\partial t}(\mathbf{E} \times \mathbf{B})
\end{aligned} .
$$

Here, $\mathbf{E}$ denotes the electric field and $\varepsilon_{0}$ is the electric permittivity of free space. This symmetric form includes both electric and magnetic field contributions, but in the case considered here the electric terms can be neglected to get the Maxwell magnetic body force as follows

$$
\mathbf{f}^{\mathrm{M}}=-\frac{1}{\mu_{0}}(\mathbf{B} \cdot \nabla) \mathbf{B}-\frac{1}{2 \mu_{0}} \nabla(\mathbf{B} \cdot \mathbf{B}),
$$

where $\nabla \cdot \mathbf{B}=0$ was exploited. The total transduction force $\mathbf{F}$ can be also written with Maxwell's stress tensor $\mathbf{T}^{\mathrm{M}}$ in the following form

$$
\mathbf{F}=\int_{V} \mathbf{f}^{\mathrm{M}} d V=\int_{S} \mathbf{T}^{\mathrm{M}} \cdot \mathbf{n} d S .
$$


Maxwell's stress tensor is symmetric and, in the absence of significant electric field, it can be related to Maxwell's body force via $\mathbf{f}^{\mathrm{M}}=\nabla \cdot \mathbf{T}^{\mathrm{M}}$. In terms of the magnetic flux density, Maxwell's stress tensor can be written as follows

$$
\mathbf{T}^{\mathrm{M}}=\frac{1}{\mu_{0}}\left[(\mathbf{n} \cdot \mathbf{B}) \mathbf{B}-\frac{1}{2} \mathbf{n}(\mathbf{B} \cdot \mathbf{B})\right] .
$$

It should be mentioned that Brown has also derived this form of the external force as one of the equivalent representations of the total transduction force (see Eq. 2.41 on page 20 of [5]). Then, the total transduction force can be calculated by integrating this stress tensor over the whole surface of the body. In a computational implementation of this method the integration can be often limited to the surface of the body directly below the EMAT since the stress tensor vanishes everywhere else. Also, care must be taken to take the magnetic flux density, or at least its tangential component, inside the body since it is discontinuous at the surface.

In summary, Table 1 lists the above described four methods that can be used to calculate the total transduction force of EMATs (in the absence of magnetostrictive effects). For consistency, the notation $\mathbf{T}^{\mathrm{m} 4}=\mathbf{T}^{\mathrm{M}}$ was introduced for Maxwell's stress tensor, while $\mathbf{f}^{\mathrm{L} 4}=\mathbf{f}^{\mathrm{m} 4}=\mathbf{0}$. Using the above introduced body force and surface traction components, the total transduction force of the $i$ th method $(i=1,2,3,4)$ can be calculated from

$$
\mathbf{F}=\int_{V} \mathbf{f}^{\mathrm{L} i} d V+\int_{V} \mathbf{f}^{\mathrm{m} i} d V+\int_{S} \mathbf{T}^{\mathrm{m} i} d S
$$

It is probably the easiest to calculate $\mathbf{f}^{\mathrm{L} 1}$ and $\mathbf{f}^{\mathrm{m} 1}$ directly based on the external magnetic field $\mathbf{H}^{\mathrm{e}}$ since it is non-rotational therefore both interpretations of the tensor gradient yield the same result and the surface traction vanishes. However, proper care must be taken when the transduction force is calculated from the total magnetic field. The safest option is to use Brown's original formuli of method \#2 with the body forces $\mathbf{f}^{\mathrm{L} 2}$ and $\mathbf{f}^{\mathrm{m} 2}$ calculated from the total magnetic field $\mathbf{H}$. Another option is to use the alternative formuli of method \#3 with the body forces $\mathbf{f}^{\mathrm{L} 3}$ and $\mathbf{f}^{\mathrm{m} 3}$ calculated from the total magnetic flux density $\mathbf{B}$ and total magnetic field $\mathbf{H}$, respectively. The non-vanishing surface traction $\mathbf{T}^{\mathrm{m} 2}=\mathbf{T}^{\mathrm{m} 3}$ is calculated from the normal component of the magnetization $M_{\mathrm{n}}$ using Eq. (15) for both methods \#2 and \#3. The benchmark method based on Maxwell's stress tensor is listed as method \#4. 
Table 1: List of methods that can be used to calculate the total transduction force of EMATs. The parentheses refer two the two different interpretations of the gradient of a vector described in the text, namely $\mathbf{M} \cdot(\nabla \mathbf{H})=M_{j} H_{j, i} \hat{e}_{i}$ and $(\mathbf{M} \cdot \nabla) \mathbf{H}=M_{j} H_{i, j} \hat{e}_{i}$.

\begin{tabular}{|c|c|c|c|}
\hline method & $\mathbf{f}^{\mathrm{L}}$ & $\mathbf{f}^{\mathrm{m}}$ & $\mathbf{T}^{\mathrm{m}}$ \\
\hline 1 & $\mu_{0} \mathbf{J}^{\mathrm{c}} \times \mathbf{H}^{\mathrm{e}}$ & $\mu_{0}(\mathbf{M} \cdot \nabla) \mathbf{H}^{\mathbf{e}}$ & none \\
\hline 2 & $\mu_{0} \mathbf{J}^{\mathrm{c}} \times \mathbf{H}$ & $\mu_{0}(\mathbf{M} \cdot \nabla) \mathbf{H}$ & $\frac{1}{2} \mu_{0} M_{\mathrm{n}}^{2} \mathbf{n}$ \\
\hline 3 & $\mathbf{J}^{\mathrm{c}} \times \mathbf{B}$ & $\mu_{0} \mathbf{M} \cdot(\nabla \mathbf{H})$ & $\frac{1}{2} \mu_{0} M_{\mathrm{n}}^{2} \mathbf{n}$ \\
\hline 4 & none & none & $\frac{1}{\mu_{0}}\left[(\mathbf{n} \cdot \mathbf{B}) \mathbf{B}-\frac{1}{2} \mathbf{n}(\mathbf{B} \cdot \mathbf{B})\right]$ \\
\hline
\end{tabular}

It is interesting to examine which of the above listed methods are the most popular in the literature available on computational modeling of EMATs. Method \#1 which requires the separate assessment of the magnetic field produced by the EMAT itself in the absence of the conducting ferromagnetic body has rarely been applied directly for modeling of EMATs, although separate calculation of $\mathbf{H}^{\mathrm{e}}$ is hardly a difficult task compared to the mapping of the eddy current density $\mathbf{J}^{\mathrm{c}}$ and magnetization $\mathbf{M}$ distributions. Methods \#2 and \#3 are more widely used following the seminal works of Thompson [7,12] and Ogi [17,18], respectively. Wilbrand's work in the 1980's [19,20] built on Thompson's groundbreaking efforts and continued to use $\mathbf{f}^{\mathrm{m} 2}$ for the magnetization force, but in combination with $\mathbf{f}^{\mathrm{L} 3}$, a questionable choice that was also adapted later in Thompson's widely used review of EMAT's published in the popular series entitled Physical Acoustics [21]. Jafari-Shapoorabadi et al. [22] and Rouge et al. [23-25] followed essentially the same approach of calculating the total Lorentz body force according to $\mathbf{f}^{\mathrm{L} 3}$ while combining it with the magnetization force $\mathbf{f}^{\mathrm{m} 2}$ and $\mathbf{f}^{\mathrm{m} 3}$, respectively, but without any surface traction component. Ludwig [26,27] and more lately Ashigwuike et al. [28] used $\mathbf{f}^{\mathrm{L} 2}$ in combination with $\mathbf{f}^{\mathrm{m} 3}$ and also omitted the surface traction term. Finally, in a recent paper Seher 
et al. also combined the total Lorentz body force $\mathbf{f}^{\mathrm{L} 3}$ with the magnetization force $\mathbf{f}^{\mathrm{m} 2}$ and surface traction $\mathbf{T}^{\mathrm{m} 2}=\mathbf{T}^{\mathrm{m} 3}$ to study the mode selectivity of Lamb-type guided waves in ferromagnetic plates [29]. Whether or not these models provide good approximations of the EMAT's performance depends on the type of EMAT configuration and the properties of the ferromagnetic material the EMAT is applied on. However, as the numerical simulation results to be presented in the next section will illustrate, the discrepancy between these approximations and the proper transduction equations based on the total magnetic field according to methods \#2, \#3, and \#4 could be quite significant.

\section{Numerical Simulation}

In ferromagnetic materials magnetization is a highly nonlinear hysteretic phenomenon that exhibits remanence, coercivity, hysteresis, and a variety of other complex features including magnetostriction. These effects are very difficult to model accurately, if for nothing else, because of the sheer number of uncertain material parameters involved. In the following numerical simulations what could be called a strongly paramagnetic material model will be used that assumes that the magnetization $\mathbf{M}=\chi \mathbf{H}$ is linearly proportional to the magnetic field $\mathbf{H}$, where $\chi$ denotes the magnetic susceptibility. This simplistic linear magnetization model is identical to the one recently used by Rouge et al. [23,24] and Seher et al. [29] and is well suited to create benchmark problems for the comparison of different EMAT models. In the following the relative permeability $\mu_{\mathrm{r}}=1+\chi$ will be used as the principal material parameter to characterize the ability of the material to magnetically polarize and the magnetic flux density will be written as $\mathbf{B}=\mu_{0} \mu_{\mathrm{r}} \mathbf{H}$.

In the strongly paramagnetic model the magnetization $\mathbf{M}$ can be calculated from the magnetic field $\mathbf{H}$ or the magnetic flux density $\mathbf{B}$ from the following equation

$$
\mathbf{M}=\left(\mu_{\mathrm{r}}-1\right) \mathbf{H}=\frac{\mu_{\mathrm{r}}-1}{\mu_{0} \mu_{\mathrm{r}}} \mathbf{B}
$$

The normal surface traction given in Eq. (15) can be re-written as

$$
\mathbf{T}^{\mathrm{m} 2}=\frac{\left(\mu_{\mathrm{r}}-1\right)^{2}}{2 \mu_{0} \mu_{\mathrm{r}}^{2}} B_{\mathrm{n}}^{2} \mathbf{n},
$$


which is computational more convenient than the original form because the normal component of the magnetic flux density $B_{\mathrm{n}}$ is continuous throughout the interface on which the surface traction must be calculated.

The following Finite Element (FE) numerical simulations were conducted using the commercially available FE software COMSOL Multiphysics [30] that lends itself quite readily to modelling coupled electromagnetic and elasticity problems. The modelling approach follows the previous work of Ribichini et al. [3] and Seher et al. [29] where FE models were developed for shear and Lamb wave EMAT applications. Two types of EMAT configurations will be considered in the numerical simulations, a longitudinal wave and a shear wave EMAT, and the various transduction methods presented earlier will be compared to each other. The aim of this comparison is to clarify the proper governing equations for the electromagnetic-elastic interaction, while further analysis of the performance of these two EMATs selected for illustration purposes only is beyond the scope of this paper.

\subsection{Model Overview}

Figure 1 (a) and (b) show schematic views of the axisymmetric EMAT models chosen to illustrate longitudinal and shear wave generation, respectively. The longitudinal wave EMAT model features a bias magnetic field that is essentially parallel to the specimen surface within the skin depth layer as indicated by an arrow in the in Figure 1(a). The bias field is produced by an inner and an outer magnet of opposite polarization with a flux guide of high magnetic permeability at the top to guide the magnetic flux on the return path. The induction coil is modelled as a ten-turn pancake spiral coil that is located between the two magnets and driven by a 1 A alternating current. The geometric dimensions of the EMAT are listed in Table 2, where magnet 1 is the inner magnet and magnet 2 is the outer one representing a ring due to the axisymmetric nature of the model. The shear wave EMAT model differs from the longitudinal wave EMAT only in terms of the magnet arrangement, whereby the outer magnet and the flux guide are omitted and the diameter of the inner magnet is increased such that the bias magnetic field in the skin depth layer is essentially orthogonal to the specimen surface as indicated by the arrow in Figure 1(b). The geometric dimensions of the shear wave EMAT model are also listed in Table 2. 
The specimen is modelled as a cylinder with a length of $400 \mathrm{~mm}$ and a diameter of $60 \mathrm{~mm}$. For modelling the wave propagation, the outer wall assumes roller boundary condition in case of the longitudinal EMAT model and free boundary condition in the shear wave model EMAT. In the latter case, the choice of free boundary condition is somewhat arbitrary, but has no effect on the transduction sensitivity of the EMAT that is the main concern in this paper. The "bottom" of the cylinder for both models is terminated with a low-reflecting boundary in order to avoid reverberations in the specimen as the simulations are run in the frequency domain. The magnets are surrounded by a sufficiently large air domain in order to model the return path of the magnetic flux. All domains are meshed fine enough to minimize spatial discretization errors. Special emphasis is placed on sufficiently fine discretization of the sub-surface region where the electromagnetic-acoustic interaction takes place.

Table 2: Geometric dimensions of the longitudinal and shear wave EMAT FE models.

\begin{tabular}{|c|c|c|}
\hline Dimension [mm] & Longitudinal wave EMAT & Shear wave EMAT \\
\hline Inner coil diameter & 14 & 26 \\
\hline Outer coil diameter & 26 & 30 \\
\hline Magnet 1 diameter & 10 & n. a. \\
\hline Magnet 2 outer diameter & 30 & n. a. \\
\hline Magnet 2 inner diameter & 41.2 & 20 \\
\hline Magnet 1 \& 2 height & 20 & n. a. \\
\hline Flux guide height & 5 & \\
\hline
\end{tabular}

The electromagnetic material parameters for the permanent magnet assume those of an N42 grade Neodymium magnet with remnant magnetic flux density of $1.3 \mathrm{~T}$, relative magnetic permeability of 1.05 (recoil permeability), and electric conductivity of $0.8 \mathrm{MS} / \mathrm{m}$. The magnetic flux guide exhibits a significantly higher relative magnetic permeability of 1000 and zero electric conductivity. For the steel specimen, electric conductivity of $4.032 \mathrm{MS} / \mathrm{m}$ is assumed and the relative magnetic permeability is left as a variable in the following parametric study. The reason for this is that in the case of the strong paramagnetic approximation, as made here, the relative magnetic permeability allows for the variation of the ratio between the Lorentz and 
magnetization force contributions. Alternatively, the electric conductivity could have been varied, however, in practice, the relative magnetic permeability is subject to significantly larger variations than the electric conductivity, for which reason the magnetic permeability is selected as the parameter of study. The elastic properties of the specimen were selected to represent a typical soft steel with density of $7850 \mathrm{~kg} / \mathrm{m}^{3}$, Young's modulus of $200 \mathrm{GPa}$, and Poisson ratio of 0.33 .

The elastic domain is discretized with a maximum element size of $1 / 30$ of the elastic wave length of the slowest (shear) wave. The excitation of the elastic domain is applied only in an interaction region of limited depth representing at least ten times the standard electromagnetic penetration depth, since the dynamic electric and magnetic fields are negligible elsewhere. Due to the large ratio between the elastic wave length and the electromagnetic skin depth, the interaction region is discretized with higher meshing density that increases in the boundary layer below the surface.

The aim of the following simulations is the numerical comparison of the absolute sensitivity of EMAT transmitters modeled by various transduction methods listed in Table 1. Transduction sensitivity will be measured as the average particle velocity in the principal polarization direction at a given distance from the EMAT. The sensitivity will be plotted as a function of the magnetic permeability at $5 \mathrm{kHz}$ and $50 \mathrm{kHz}$. Although very few EMATs are used below $20 \mathrm{kHz}$, the 5 $\mathrm{kHz}$ results are included in order to demonstrate that method \#1 based on the external field only asymptotically approaches the full field solutions obtained by methods \#2, \#3, and \#4 when $\delta<<\lambda$. In addition, a limited number of simulation results will be shown for the radially polarized shear wave transducer at $1 \mathrm{MHz}$.

\subsection{Longitudinal Wave EMAT Results}

Figure 2 shows the results obtained for the longitudinal EMAT using the four transduction methods listed in Table 1. The longitudinal wave particle velocity magnitude is plotted as a function of the relative magnetic permeability for $5 \mathrm{kHz}$ and $50 \mathrm{kHz}$ in Figure 2(a) and 2(b), respectively. All four methods yield the same results for a relative magnetic permeability of unity, corresponding to the "non-magnetic" case, for which only the Lorentz force is present while the magnetization in the specimen vanishes. The curves for methods \#2, \#3 and \#4 line up on top of each other for both frequencies, implying that these methods yield the same results for 
the EMAT configuration considered here. In terms of method \#2 and \#3, this comes as no surprise since it has been shown earlier in Equation (22) that these methods are equivalent. Furthermore, since method \#4 also yields the same results as methods \#2 and \#3, one conclusion is that the separation into Lorentz and magnetization force is arbitrary and determined by the choice of equations for the transduction force. Also, methods \#2, \#3 and \#4 exhibit equally good approximations of the external and internal transduction forces. When comparing method \#1 with the other three methods, it can be observed from Figure 2 that the discrepancy is small for the $5 \mathrm{kHz}$ case and larger for the $50 \mathrm{kHz}$ case, confirming the argument which has been made earlier for similar results in the low-frequency approximation. The discrepancy increases with frequency as the spatial distribution of the transduction forces starts to exhibit its influence, however still leading to qualitatively similar results. Therefore, from a practical modelling point of view, method \#1 may yield a sufficiently accurate approximation for low frequency regimes. However, since it neglects the contribution of internal forces, it may lead to an erroneous approximation when the interaction zone is not entirely negligible with respect to the acoustic wavelength.

A great variety of equations have been used in the literature by various authors to model the transduction forces produced by various EMATs, which deviate from methods \#1-4 presented in this paper as the correct alternatives. Therefore, it is necessary to study how combinations of various Lorentz and magnetization forces as well as surface traction terms influence the absolute sensitivity of EMATs in different scenarios. The reference case is chosen to be method \#2, which will be used as a benchmark method. The various cases to be considered in this section are listed in Table 3 and the respective simulation results are shown in Figure 3 (case 1-5) and Figure 4 (case 6-7), again giving the longitudinal wave particle velocity magnitude as a function of the magnetic permeability for $5 \mathrm{kHz}$ in (a) and $50 \mathrm{kHz}$ in (b).

The first observation form Figure 3 is that when considering only the body forces, as in cases 1 and 2, the longitudinal wave particle velocity magnitude monotonically approaches zero. This observation has led to the conclusion in literature [2,18] that the Lorentz and magnetization forces cancel each other for EMAT configurations like the one considered here. From Figure 3 it can be also seen that neglecting the surface traction term, as for example found in [22-28], leads to a good approximation of the transduction force only for a very low relative magnetic permeability, when the surface traction term diminishes compared to the sum of the bulk forces. 
In contrast, considering the surface traction term only (case 3) yields a good approximation of the transduction force for high relative magnetic permeability. The combination of Lorentz and magnetization force terms of method \#2 and \#3 is also illustrated in Figure 3 (cases 4-5). This approach can be also found in literature, for example in [22-25], where the surface traction is neglected, and [29], where it is not. The comparison of cases 4-5 with the reference case shows that there is a significant discrepancy in longitudinal wave particle velocity magnitude, especially for low values of magnetic permeability.

Table 3: Overview various combinations of Lorentz and magnetization forces as well as surface traction for the longitudinal wave EMAT simulations. The various transduction terms are listed in Table 1.

\begin{tabular}{|c|c|c|c|}
\hline case & $\mathbf{f}^{\mathrm{L}}$ & $\mathbf{f}^{\mathrm{m}}$ & $\mathbf{T}^{\mathrm{m}}$ \\
\hline reference & $\mathbf{f}^{\mathrm{L} 2}$ & $\mathbf{f}^{\mathrm{m} 2}$ & $\mathbf{T}^{\mathrm{m} 2}$ \\
\hline 1 & $\mathbf{f}^{\mathrm{L} 2}$ & $\mathbf{f}^{\mathrm{m} 2}$ & $\mathbf{0}$ \\
\hline 2 & $\mathbf{f}^{\mathrm{L} 3}$ & $\mathbf{f}^{\mathrm{m} 3}$ & $\mathbf{0}$ \\
\hline 3 & $\mathbf{0}$ & $\mathbf{0}$ & $\mathbf{T}^{\mathrm{m} 2}$ \\
\hline 4 & $\mathbf{f}^{\mathrm{L} 2}$ & $\mathbf{f}^{\mathrm{m} 3}$ & $\mathbf{T}^{\mathrm{m} 2}$ \\
\hline 5 & $\mathbf{f}^{\mathrm{L} 3}$ & $\mathbf{f}^{\mathrm{m} 2}$ & $\mathbf{T}^{\mathrm{m} 2 *}$ \\
\hline 6 & $\mathbf{f}^{\mathrm{L} 2}$ & $\mathbf{f}^{\mathrm{m} 2 \dagger}$ & $\mathbf{T}^{\mathrm{m} 2^{*}}$ \\
\hline 7 & $\mathbf{f}^{\mathrm{L} 2}$ & $\mathbf{f}^{\mathrm{m} 2 \dagger}$ & $\mathbf{T}^{\mathrm{m} 2 *}$ \\
\hline
\end{tabular}

Figure 4 compares cases 6-7 to the respective reference case for (a) $5 \mathrm{kHz}$ and (b) $50 \mathrm{kHz}$. The motivation for these cases is that in ferromagnetic materials the static magnetization may be significantly larger than the dynamic magnetization $\left(\mathbf{M}_{\text {static }}>>\mathbf{M}_{\text {dynamic }}\right)$. This is implemented in the FE model by removing all terms in the body force equations that contain the dynamic magnetization. From Figure 4 it can be seen, that disregarding the terms containing the dynamic magnetization leads to a good approximation of the reference case, for both methods \#2 and \#3 over the whole range of relative magnetic permeability. The largest discrepancy between the 
curves occurs at lower values of magnetic permeability (between approximately 5 and 50) and the approximation is better at $50 \mathrm{kHz}$. It needs to be noted that this observation applies to the EMAT configuration considered in this section and may lead to different results for other EMAT configurations.

\subsection{Shear Wave EMAT Results}

Figure 5 shows the comparison of the various transduction force methods from Table 1 for the chosen shear wave EMAT configuration at (a) $5 \mathrm{kHz}$ and (b) $50 \mathrm{kHz}$. Since this EMAT configuration generates radially polarized shear waves, the transverse particle velocity magnitude is chosen as the absolute measure of transduction sensitivity as a function of relative magnetic permeability. From Figure 5, it can be seen that methods \#2-\#4 show very good agreement, with methods \#2 and \#3 yielding identical results as expected. This result confirms the previous finding that the separation of the transduction force into Lorentz and magnetization components is indeed arbitrary and the outcome does not depend on whether method \#2 or \#3 is chosen. Similarly to the longitudinal EMAT simulations, method \#1 leads to a good approximation of the transduction force at $5 \mathrm{kHz}$ and less so at $50 \mathrm{kHz}$. It should be mentioned that in this case there is a very small but perceivable discrepancy between methods \#2 and \#3 on one side and method \#4 on the other side at $50 \mathrm{kHz}$. This minor discrepancy is due to the fact that method \#4 attributes the total transduction force to a surface traction which has a very strong tangential component while the surface traction term in both methods \#2 and \#3 is strictly normal to the surface.

In typical NDT applications, such as thickness gauging, radially polarized shear wave EMATs are often operated in the MHz range, where the $\delta / \lambda$ ratio is significantly higher than at the lower frequencies considered earlier. Therefore, a parametric study was also conducted at 1 MHz operation frequency and the results obtained by methods \#1-\#4 are shown in Figure 6. For this study, the model was modified slightly such that its dimensions are scaled down by a factor of 20 relative to the $50 \mathrm{kHz}$ case, in order to reduce the computational effort and the specimen diameter (including the air domain) were proportionally increased by a factor of 1.5 after scaling. The results are similar to the lower frequency simulation results, where methods \#2-\#4 show very good agreement with each other over the whole permeability range, but only for very low relative magnetic permeability with method \#1. The slight discrepancy between method \#4 and \#2-\#3 at low magnetic permeability is attributed to difficulties encountered in modelling the 
interaction zone. There, the electromagnetic skin depth is small but not completely negligible with respect to the acoustic wavelength and the modeled depth of the interaction zone, indicating that the depth of the interaction zone for the body forces must be increased in simulations at higher operation frequencies.

As a final example, Figure 7 shows cases 1, 4, and 5 from Table 3 compared to the correct reference result obtained using method \#2 for the shear wave EMAT configuration. From Figure 7 it can be seen that at $5 \mathrm{kHz}$ neglecting the surface traction force leads to a similar result as found for the longitudinal wave EMAT configuration, for which the wave velocity magnitude also decreases towards zero for increasing relative magnetic permeability. At $50 \mathrm{kHz}$, neglecting the surface traction leads to fairly good agreement with the correct solution over a large range of magnetic permeability (up to a value of approximately 100). When mixing the Lorentz and magnetization force terms of methods \#2 and \#3, the results show a significant discrepancy with the correct solution, over the whole range of relative magnetic permeability at both frequencies. Similar observations were also made for the $1 \mathrm{MHz}$ case, though the results are not shown here.

\section{Conclusions}

The physical interpretation of what is routinely referred to in the NDE community as the Lorentz force transduction mechanism of EMATs has been shown to be inconsistently defined in the literature. Our analysis suggested that, in connection with the other principal mechanism usually referred to as magnetization, in conducting magnetizable materials the Lorentz force cannot be uniquely defined as a specific component of the total electromagnetic transduction force. Instead, a better definition of the Lorentz force is that it is the asymptotic limit of the electromagnetic transduction force as the magnetic susceptibility of the material vanishes. Similarly, the best definition of the magnetization force is that it is the asymptotic limit of the electromagnetic transduction force as the electric conductivity of the material vanishes. In between these extreme limits, where almost all magnetizable materials fall with the possible exception of ferrites, the difference between Lorentz and magnetization mechanisms is blurred and to a large degree remains a question of semantics.

Four alternative methods were considered to compute the transduction force in EMAT applications. One method is based on the external field quantities only that disregards magnetic sources within the specimen and therefore all internal force contributions. The other three 
methods are based on the total field quantities, i.e., they account for both internal and external force contributions. For the methods based on the total field, it was shown that the separation into Lorentz and magnetization forces is somewhat arbitrary and determined by the formulation chosen for the implementation. One of the three total-field methods that can be used to benchmark all other methods is based on Maxwell's stress tensor that does not make any assumption on the separation of the electromagnetic transduction force into Lorentz and magnetization components.

Numerical simulations of longitudinal and shear wave EMAT configurations were used to compare the absolute sensitivity of EMATs modeled using various formulations encountered in the literature. It was found that all three methods based on the total magnetic field lead to a very good agreement with each other. The method based on the external magnetic field only leads to an approximation of the correct methods based on the total field, however, a significant discrepancy is observed, especially for high magnetic permeability and high inspection frequency. When combining or omitting various terms for the body forces or surface traction of methods \#2 and \#3, as often encountered in literature, the results show that such erroneous simulations can lead to significant discrepancy in comparison to the correct solution.

In connection with the presented numerical simulations, it needs to be pointed out that while some of the equations to model the transduction force found in literature may not be physically correct, they may still lead to a sufficiently good approximation that matches the experimental data sufficiently well. In this respect, the large discrepancies illustrated in Figures 3 and 7 that show the absolute sensitivity of the EMAT as a function of magnetic permeability represent worst case scenarios since both the dependent and independent variables were selected to maximize the discrepancy to be illustrated. As for EMAT modeling, numerical implementation of methods \#2, \#3, or \#4 is recommended. The interaction volume and surface should be chosen sufficiently large to achieve full coverage, especially in cases where low relative magnetic permeability is expected.

\section{Acknowledgement}

The authors acknowledge useful discussions with Hirotsugu Ogi, Alain Lhémery, Michael Lowe, and Peter Huthwaite. 


\section{References}

[1] G. Alers, "A history of EMATS," in: Review of Progress in Quantitative Nondestructive Evaluation Vol. 27 (AIP, 2008) pp. 801-808.

[2] I. V. Il'in and A. V. Kharitonov, "Theory of the EMA method of detecting Rayleigh-waves for ferromagnetic and ferrimagnetic materials," Sov. J. Nondestr. Test. 16, 549-554 (1980).

[3] R. Ribichini, P. B. Nagy and H. Ogi, "The impact of magnetostriction on the transduction of normal bias field EMATs," NDT\&E Intern. 51, 8-15 (2012).

[4] W. F. Brown, "Irreversible magnetic effects of stress," Rev. Mod. Phys. 25, 131-135 (1953).

[5] W. F Brown, Magnetoelastic Interactions (Springer-Verlag, 1966).

[6] F. Moon, Magneto-Solid Mechanics (John-Wiley \& Sons, 1984).

[7] R. B. Thompson, "A model for the electromagnetic generation of ultrasonic guided waves in ferromagnetic metal polycrystals," IEEE Trans. Son. Ultrason. SU-25, 7-15 (1978).

[8] R. B. Thompson, "The relationship between radiating body forces and equivalent surface stresses: Analysis and application,” J. Nondestr. Eval. 1, 79-85 (1980).

[9] C. Rouge, A. Lhémery, and D. Ségur, "Transformation of body force localized near the surface of a half-space into equivalent surface stresses," J. Acoust. Soc. Am. 134, 26392646 (2013).

[10] P. B. Nagy and R. Ribichini, "Equivalent body-force model for magnetostrictive transduction in EMATs," in: Review of Progress in Quantitative Nondestructive Evaluation Vol. 34 (AIP, 2015) pp. 1316-1325.

[11] B. Clausse and A. Lhémery, "Transformation of body force generated by non-contact sources of ultrasound in an isotropic solid of complex shape into equivalent surface stresses," Wave Motion 60, 135-148 (2016).

[12] R. B. Thompson, "Mechanisms of electromagnetic generation and detection of ultrasonic Lamb waves in iron-nickel alloy polycrystals," J. Appl. Phys. 48, 4942-4950 (1977).

[13] K. A. Athanasiou and R. M. Natoli, Introduction to Continuum Biomechanics (Morgan \& Claypool, 2009).

[14] M. Itskov, Tensor Algebra and Tensor Analysis for Engineers (Springer, 2015).

[15] Nhan Phan-Tien, Tensor notation, Understanding Viscoelasticity: An Introduction to Rheology (Springer, 2013).

[16] D. J. Griffiths, Introduction to Electrodynamics, 4th ed. (Prentice Hall, 2013).

[17] H. Ogi, "Field dependence of coupling efficiency between electromagnetic field and 
ultrasonic bulk waves," J. Appl. Phys. 82, 3940-3949 (1997).

[18] M. Hirao, H. Ogi, EMATs for Science and Industry: Noncontacting Ultrasonic Measurements (Kluwer Academic Publishers, 2003).

[19] A. Wilbrand, "EMUS-probes for bulk waves and Rayleigh waves. Model for sound field and efficiency calculations," in: New Procedures in NDT (Springer, 1983) pp. 71-80.

[20] A. Wilbrand, "Quantitative modeling and experimental analysis of the physical properties of electromagnetic ultrasonic transducers," in: Review of Progress in Quantitative Nondestructive Evaluation Vol. 7 (Plenum, 1987) pp. 671-680.

[21] R. B. Thompson, "Physical principles of measurements with EMAT transducers," in: Physical Acoustics Vol. 19 (Academic Press, 1990) pp. 157-200.

[22] R. Jafari-Shapoorabadi, A. Konrad, and A. N. Sinclair, "The governing electrodynamic equations of electromagnetic acoustic transducers," J. Appl. Phys. 97, 10E102 (2005).

[23] C. Rouge, A. Lhémery and D. Ségur, "Modal solutions for SH guided waves radiated by an EMAT in a ferromagnetic plate," J. Phys. Conf. Ser. 353, 012014 (2012).

[24] C. Rouge, A. Lhémery, and C. Aristégui, "EMAT generation of bulk forces in a ferromagnetic plate and their equivalent surface stresses," J. Phys. Conf. Ser. 498, 012013 (2014).

[25] C. Rouge, A. Lhémery, C. Aristégui, and H. Walaszek, "Calculation of ultrasonic fields radiated in a ferromagnetic medium by an EMAT of arbitrary bias field driven by a current of arbitrary intensity," in: Review of Progress in Quantitative Nondestructive Evaluation Vol. 33 (AIP, 2014) pp. 450-457.

[26] R. Ludwig, "Theoretical basis for a unified conservation law description of the electromagnetic acoustic transduction process," IEEE Trans. Ultras. Ferr. Freq. Contr. 39, 476-480 (1992).

[27] R. Ludwig, "Numerical implementation and model predictions of a unified conservation law description of the electromagnetic acoustic transduction process," IEEE Trans. Ultras. Ferr. Freq. Contr. 39, 481-488 (1992).

[28] E. C. Ashigwuike, O. J. Ushie, R. Mackay and W. Balachandran, "A study of the transduction mechanisms of electromagnetic acoustic transducers (EMATs) on pipe steel materials," Sensors and Actuators A: Physical 229, 154-165 (2015).

[29] M. Seher, P. Huthwaite, M. J. S. Lowe, and·P. B. Nagy, "Model-based design of low frequency Lamb wave EMATs for mode selectivity," J. Nondestruct. Eval. 34, 22 (2015).

[30] COMSOL Multiphysics, COMSOL Inc., http://www.comsol.com/. 
(a)

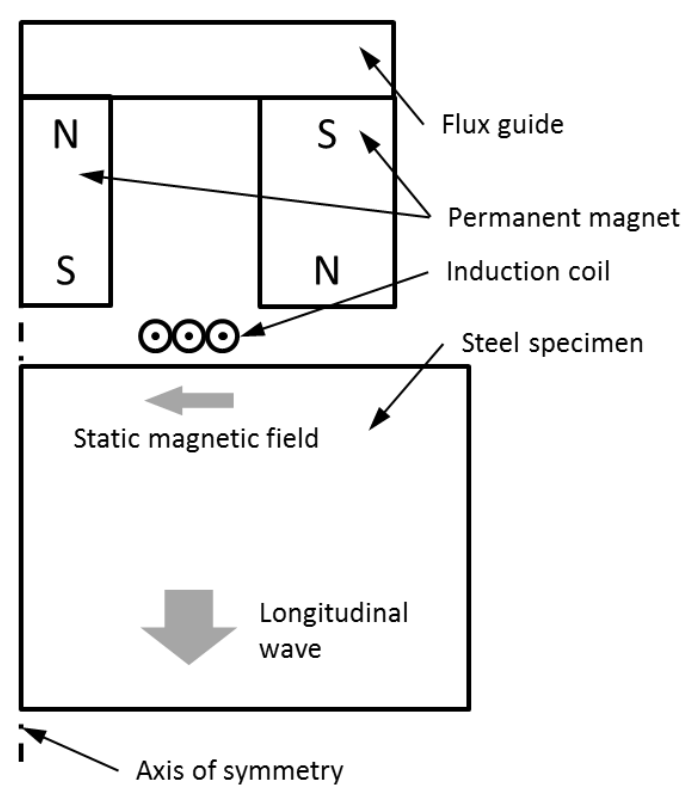

(b)

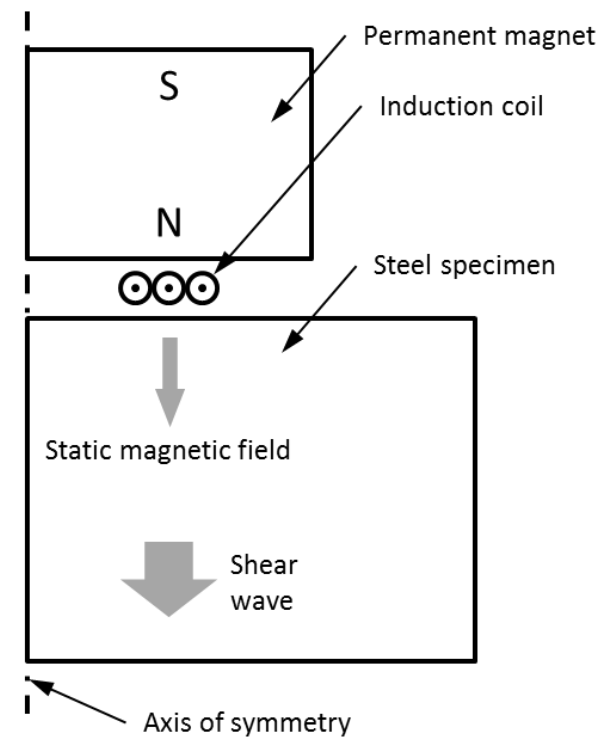

Figure 1: Overview of EMAT models showing (a) the longitudinal wave configuration and (b) the shear wave configuration. Both models are axisymmetric and only differ in the orientation of the bias magnetic field. 

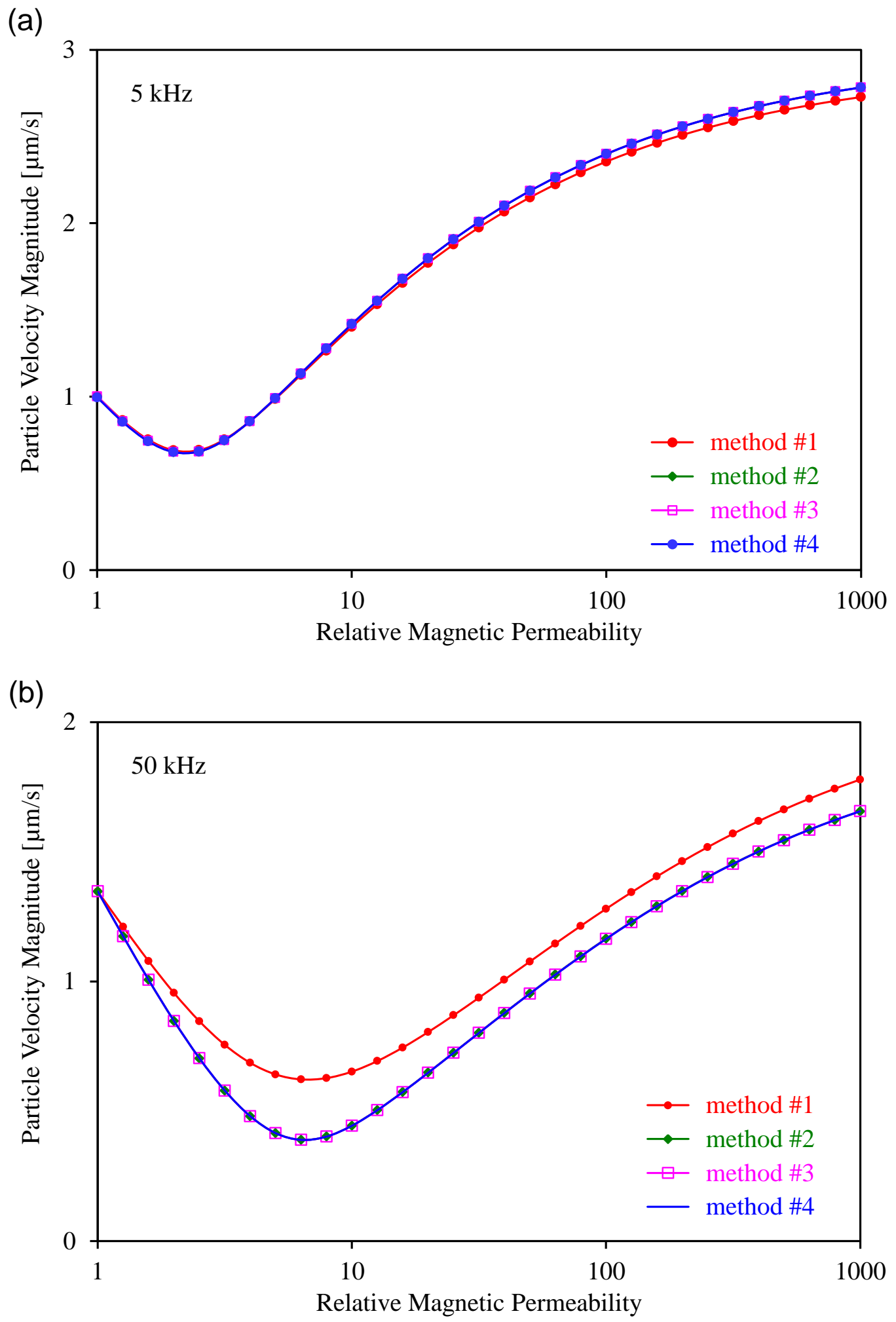

Figure 2: Longitudinal wave particle velocity magnitude as a function of the relative magnetic permeability for the longitudinal EMAT simulation (a) at $5 \mathrm{kHz}$ and (b) at $50 \mathrm{kHz}$, comparing the various transduction force calculation methods \#1 to \#4. Methods \#2-\#4 are overlapping. 

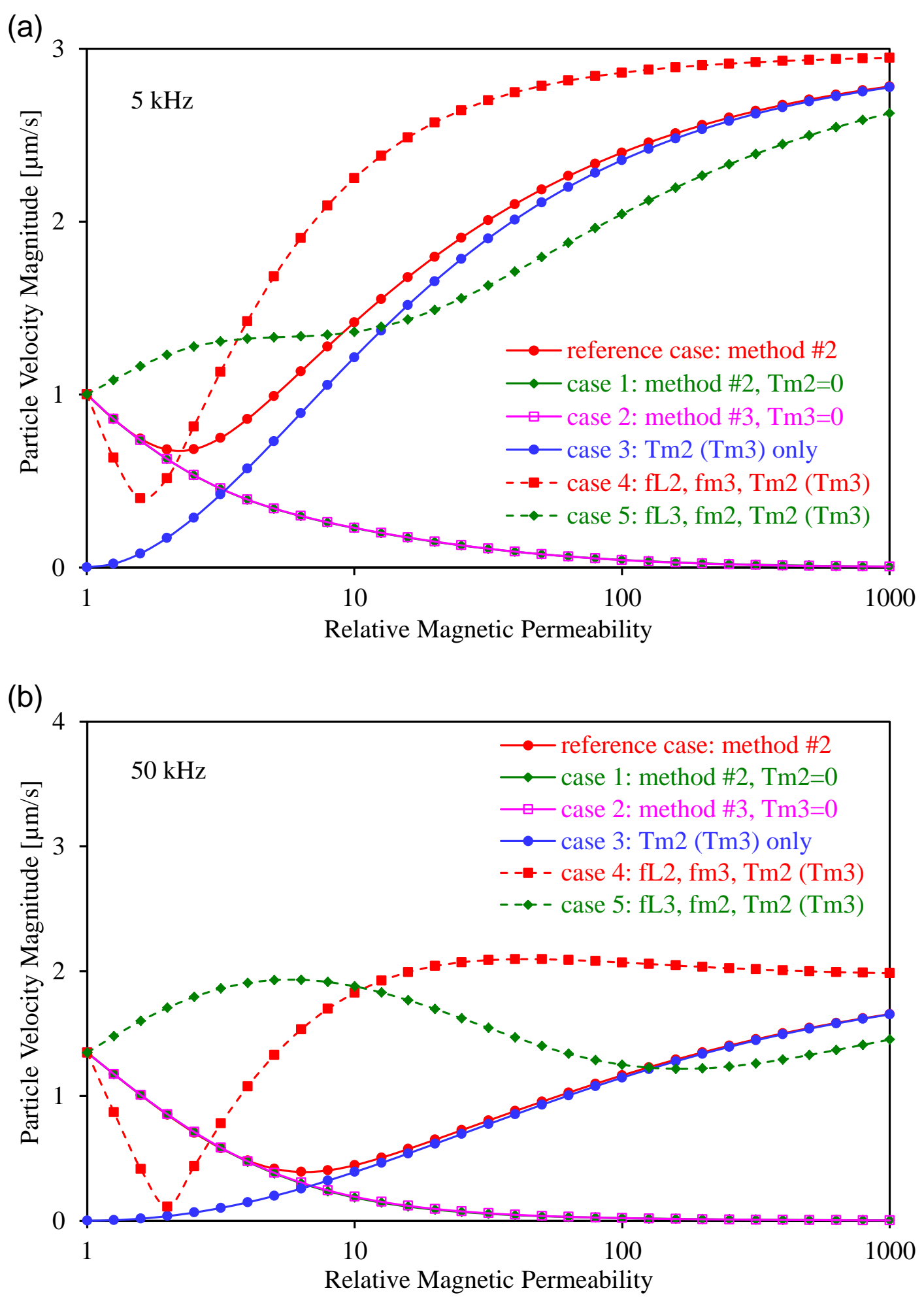

Figure 3: Longitudinal wave particle velocity magnitude as a function of the relative magnetic permeability for longitudinal EMAT simulation (a) at $5 \mathrm{kHz}$ and (b) at $50 \mathrm{kHz}$, comparing various erroneous transduction force cases, as found in literature (see Table 3). 

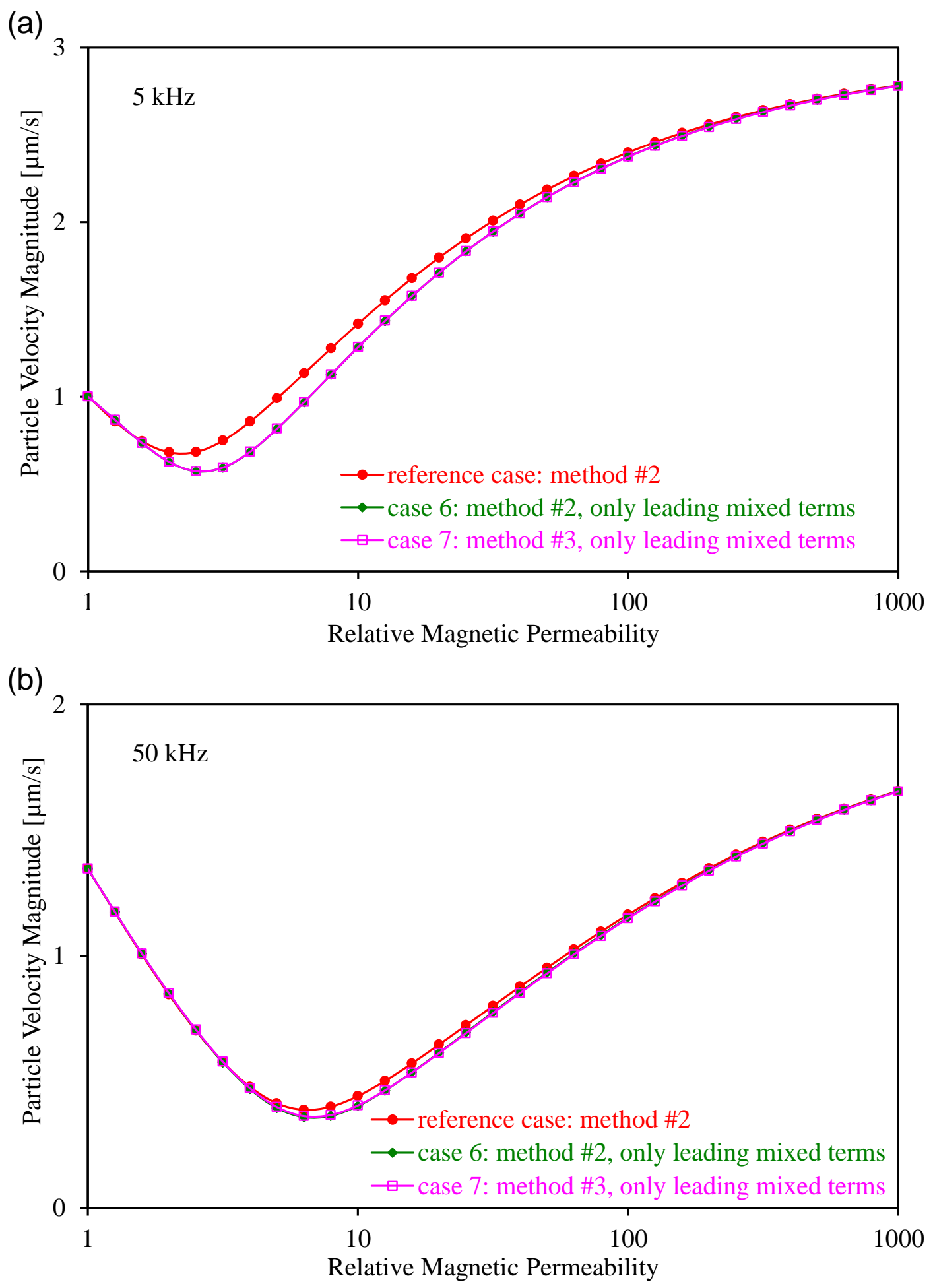

Figure 4: Longitudinal wave particle velocity magnitude as a function of the relative magnetic permeability for longitudinal EMAT simulation (a) at $5 \mathrm{kHz}$ and (b) at $50 \mathrm{kHz}$, comparing methods \#2 and \#3 when only considering the leading mixed terms (static magnetization only). 
(a)

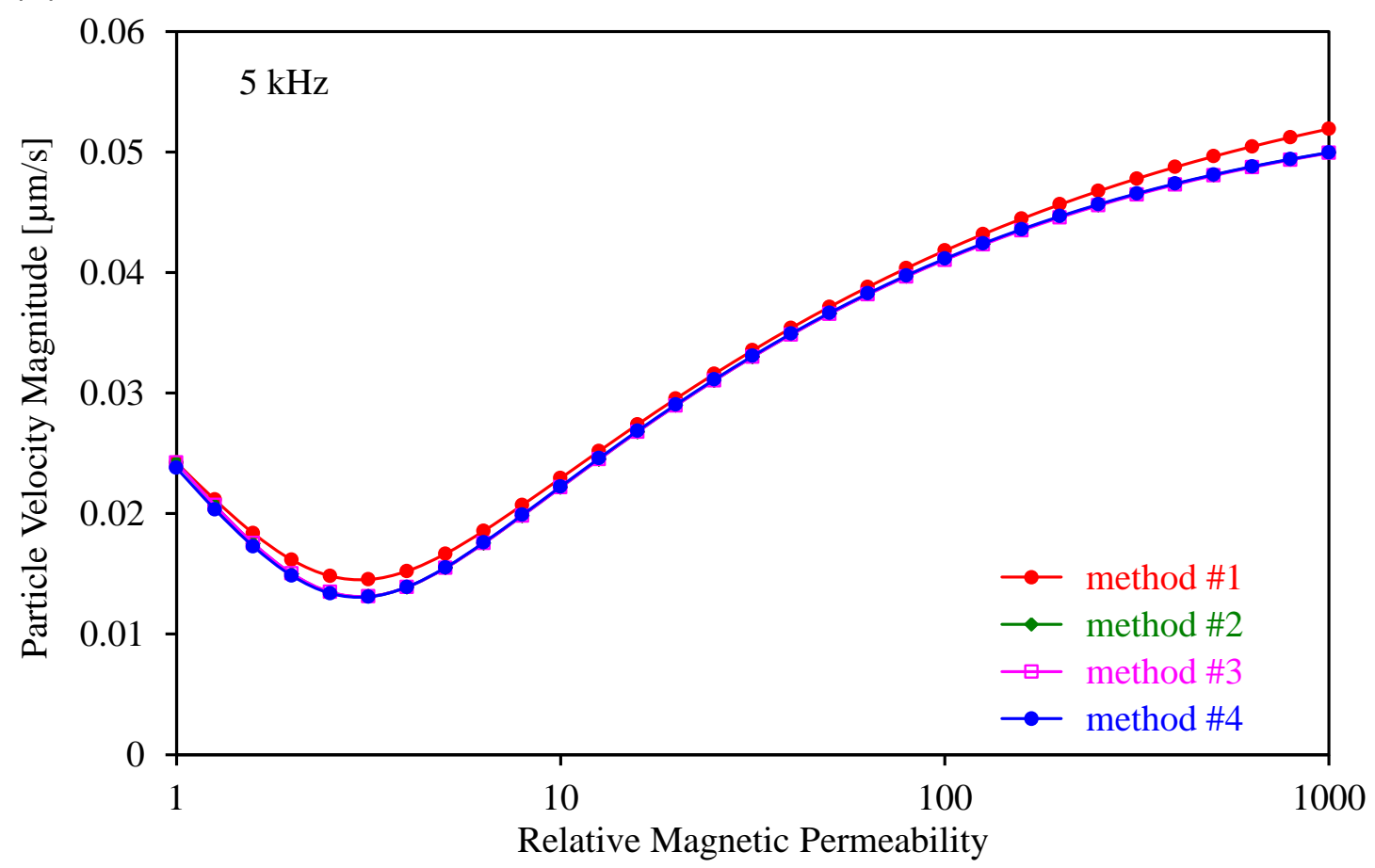

(b)

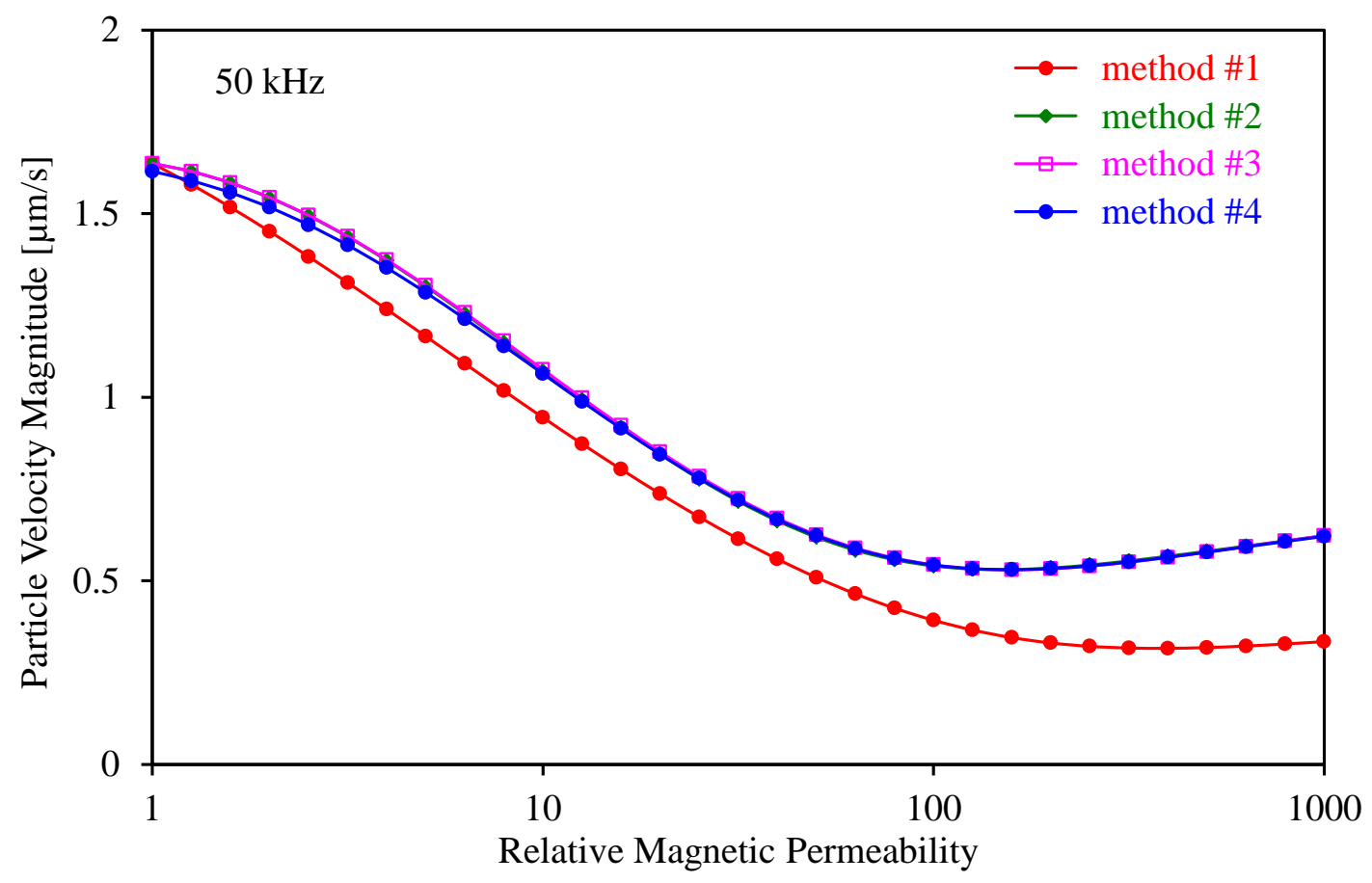

Figure 5: Shear wave particle velocity magnitude as a function of the relative magnetic permeability for the shear EMAT simulation in (a) at $5 \mathrm{kHz}$ and (b) at $50 \mathrm{kHz}$, comparing the various transduction forces (method \#1 to \#4). 


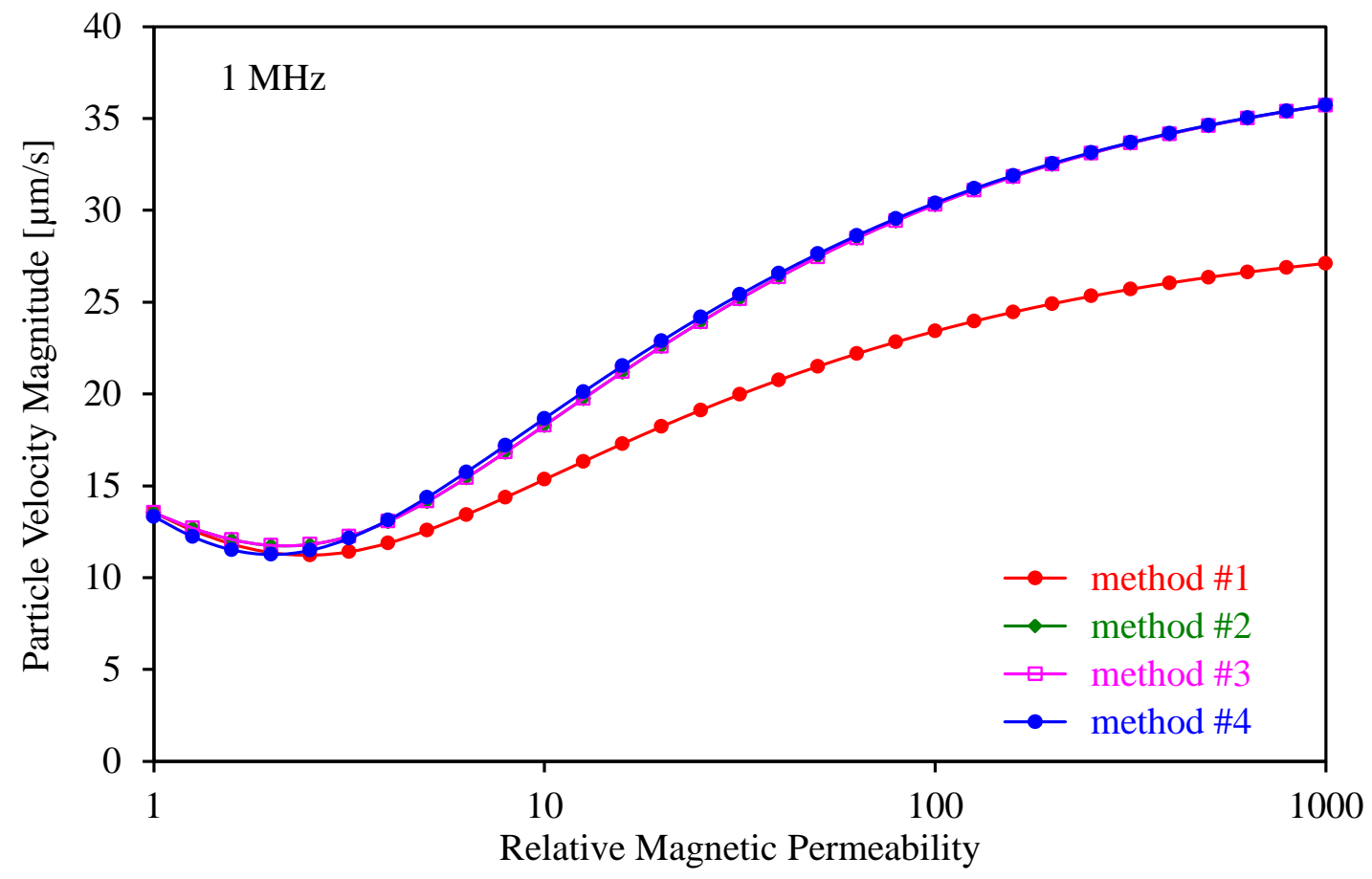

Figure 6: Shear wave particle velocity magnitude as a function of the relative magnetic permeability for the shear EMAT simulation at $1 \mathrm{MHz}$, comparing the various transduction forces (method \#1 to \#4). 
(a)

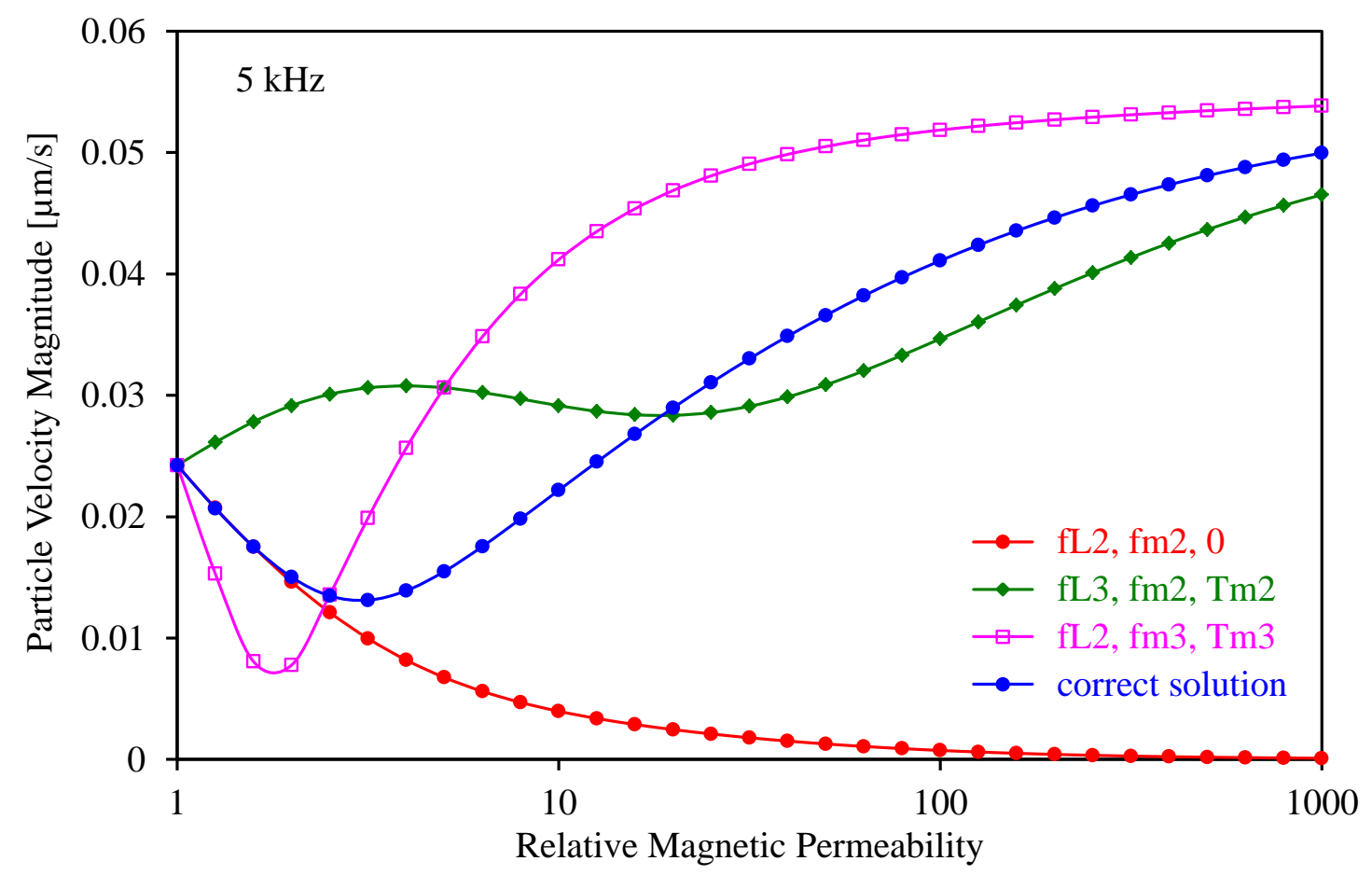

(b)

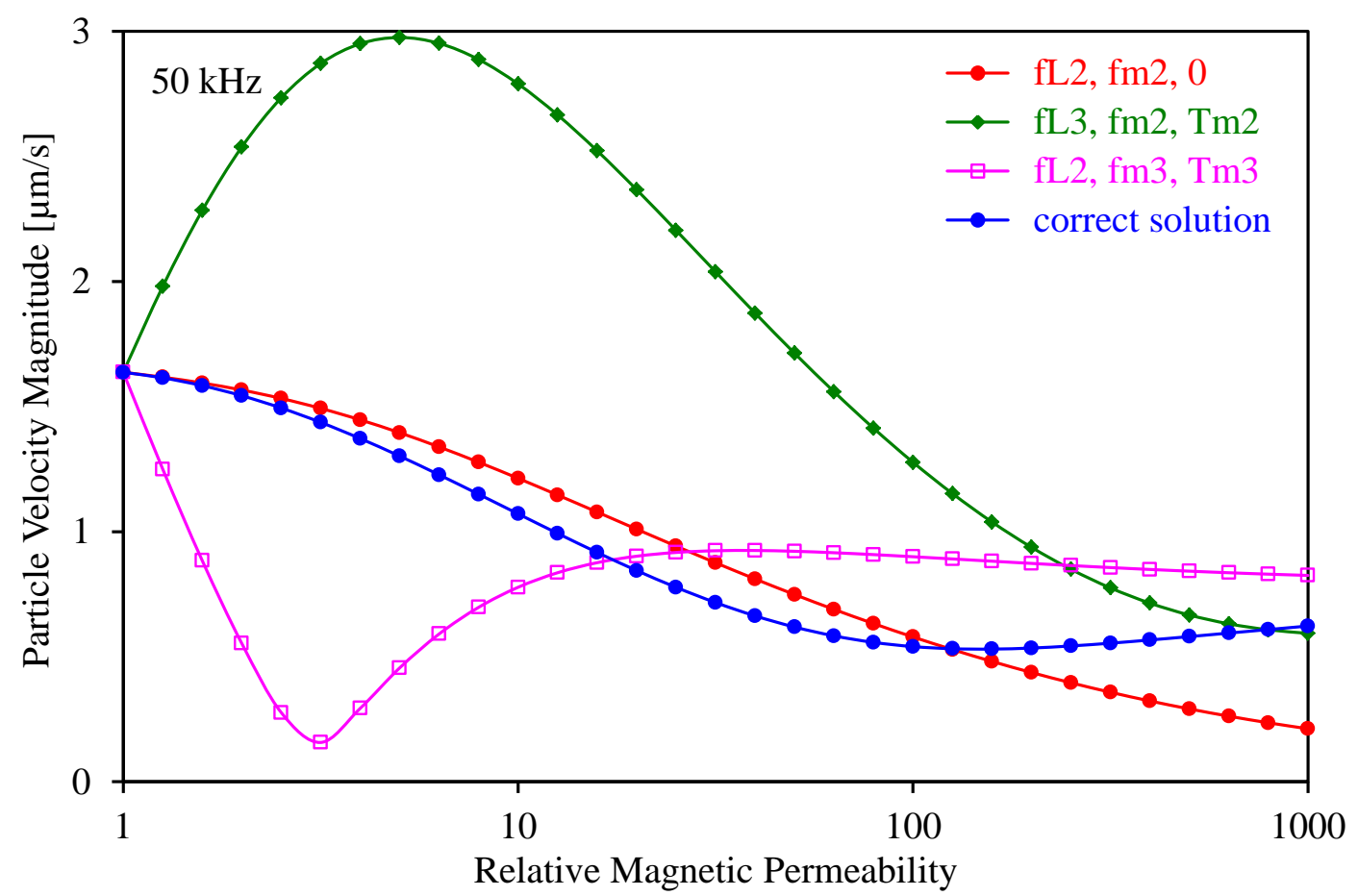

Figure 7: Shear wave particle velocity magnitude as a function of the relative magnetic permeability for the shear EMAT simulation in (a) at $5 \mathrm{kHz}$ and (b) at $50 \mathrm{kHz}$, comparing various erroneous transduction force cases, as found in literature. 